\title{
Velocity anisotropy analysis for shale lithology of the complex geological section in Jaisalmer sub-basin, India
}

\author{
Nabanita Pradhan, Saurabh Datta Gupta* (1) and P R Mohanty \\ Indian Institute of Technology (Indian School of Mines), Dhanbad, India. \\ *Corresponding author.e-mail: saurabh.doc.ism@gmail.com
}

MS received 11 December 2018; revised 8 May 2019; accepted 10 May 2019

Measurement of velocity anisotropy is an essential parameter for capturing the heterogeneity of sub-surface geology to characterise the hydrocarbon-bearing reservoir. The incorporation of velocity anisotropy parameters during the preparation of the 3D velocity model represents a robust result in a challenging geological set-up during interpretation. Generally, we can observe that the shale formation is more sensitive to velocity anisotropy response in comparison with other formations such as sandstone, siltstone for clastic reservoir or limestone and dolomite for carbonate reservoir. This study was performed mostly in the high amount shale section mixed with limestone and claystone of the Jaisalmer sub-basin area which lies in the western part of India. The preparation of the velocity model for frequent changes of lithology in the clastic and carbonate reservoir is challenging due to several changes of velocity which show a limitation in the result of the gridded velocity model. The objective of this study is to capture the changes of compressional and shear wave velocity in mixed lithology of the significant shale formation. The idea was due to the inclusion of the anisotropy incorporated changed velocity during the preparation of the gridded velocity model for correctly identified lithology. The shale formation which is the zone of investigation of the current study is situated over a carbonate sequence, and an estimated velocity anisotropy factor of this shale formation will contribute significantly during the cumulative study of velocity modelling of all formation. The current study shows that shale formation shows the character of orthorhombic anisotropy; however, this study was performed based on significant changes of well log data and related effects of vertically transverse isotropic parameters of the shale formation. The fundamental Thomsen anisotropy parameters were estimated by capturing the deviation of five independent stiffness coefficients. Significant changes in evaluated shale velocity were observed after the incorporation of the estimated Thomsen parameter in velocity values.

Keywords. P- and S-wave velocity; velocity anisotropy; compressional and shear wave velocity ratio; P-impedance; Jaisalmer sub-basin, Rajasthan; hydrocarbon exploration.

\begin{tabular}{|c|c|c|c|}
\hline \multirow{2}{*}{\multicolumn{2}{|c|}{ List of symbols }} & $P_{\mathrm{p}}$ & Pressure of pore spaces \\
\hline & & $\epsilon$ & Thomsen anisotropy parameter epsilon \\
\hline$\sigma_{i j}$ & Stress tensor & $\gamma$ & Thomsen anisotropy parameter gamma \\
\hline$m_{i j k l}$ & A stiffness tensor of rank four & $\delta$ & Thomsen anisotropy parameter delta \\
\hline$\epsilon_{k l}$ & Tensor of strain & $V_{\mathrm{P}}\left(90^{\circ}\right)$ & $\mathrm{P}$-wave in the vertical direction \\
\hline$\alpha$ & Biot's constant & $V_{\mathrm{P}}\left(0^{\circ}\right)$ & P-wave in the horizontal direction \\
\hline
\end{tabular}

Published online: 27 July 2019 
$V_{\mathrm{SH}}\left(90^{\circ}\right) \quad \mathrm{S}$-wave in the vertical direction

$V_{\mathrm{SH}}\left(0^{\circ}\right) \quad$ S-wave in the horizontal direction

$V_{i j} \quad$ Wave travelling in the axis of $X_{i}$ and polarised along the axis of $X_{j}$

$\rho \quad$ Bulk density

$V_{12} \quad$ Shear wave velocity over the axis $\left(X_{1}\right)$ and differentiated on the axis of $X_{2}$

$V_{33} \quad \mathrm{P}$-wave velocity travelling in the $X_{3}$-axis and polarised in the same axis

$V_{11} \quad$ S-wave velocity propagating along the axis $X_{1}$ and polarised in the same axis

$V_{13} \quad X$-component of $S$-wave velocity estimated from $\mathrm{P}$-wave velocity

$m_{33} \quad$ The stiffness constant in the vertical direction

$m_{66} \quad$ The stiffness constant in a plane which is horizontal that consists of shear wave in the $X_{1}$ and $X_{2}$ directions

$m_{11} \quad$ The stiffness constant in a plane where S-wave is travelling along axis $X_{1}$ and polarised in the same axis

$m_{55} \quad$ Stiffness constant in the vertical plane consisting of $\mathrm{P}$-wave along $X_{3}$ direction and S-wave along $X_{1}$ direction

$m_{13} \quad$ The stiffness constant calculated from ANNIE model

\section{Introduction}

Almost all crustal rock crusts show a certain degree of anisotropic behaviour. The anisotropic medium has a directional dependency property, i.e., the physical property of the medium varies with the direction (Tatham and McCormack 1991). Sedimentary areas always show a certain extent of anisotropy. This anisotropy may arise from different factors such as alignment of grains in the same direction, differences in grain size and layering of thin zones.

Shale formation is considered as a significant part of the sedimentary rock, and approximately $75 \%$ of all sedimentary rock comprises shale (Hornby 1994). Significant variation of seismic wave propagation governs the presence of shale formation for a sedimentary succession (Chesnokov et al. 2009). Full shale section affects seismic wave propagation and, as a consequence, distortion arises in the seismic image (Sayers 1994, 2005). The ignorance of shale anisotropy may affect adversely in various processing steps of seismic data such as NMO, DMO correction and migration.
In the gas-bearing reservoir amplitude vs. offset analysis is one of the essential steps for detecting reservoir (Ruger 2002). Negligence or avoidance of measuring of shale anisotropy parameters adversely affects the amplitude variation in the gas-bearing reservoir.

We conducted this study in the formation where the dominant lithology is shale. Grain distribution pattern shows that the orthorhombic nature is present in the shale section (Far et al. 2015); however, in our study, we observed significant changes under the vertical transverse isotropy (VTI) condition.

VTI refers to the directional characteristics of materials in which similar properties appear in all directions parallel to the planes of isotropy and different properties perpendicular to or crossing the planes of isotropy, and this perpendicular direction is known as the axis of rotational symmetry (Norris and Sinha 1993; Walsh et al. 2006). The shale section of the current study is mostly a flat-lying shale pattern with different horizontal and vertical velocities and produces faster velocity along the bed and slower velocity across the bed (Vernik and Liu 1997).

We conducted this study in an old drilled exploratory well in the Western Rajasthan area, drilled in 1986 (figure 1). We found this study well drilled in the highly faulted area of Jaisalmer subbasin. Gas discovery was observed from surrounding fault blocks. We restricted our study to the Upper Goru formation of the Late Cretaceous age where significant shale section is present. Four zones were tested in this well during the exploration. All zones were concluded to be dry or water-bearing whereas in one zone at the Lower Goru formation produced a mild show of gas. The study zone selected was based on potentiality formation, identified from the well-logging signature. We observed that the tested zone-IV $(1095-1100 \mathrm{~m})$ of the Upper Goru formation is most encouraging with respect to other tested zones and our current zone of investigation (955.0912-990.9052 m) was also restricted to zoneIV. The study well comprises only compressional sonic (P-sonic), density (Rhob) well log data for anisotropy analysis. We used several empirical relations to get the shear wave (S-wave) velocity information from compressional wave (P-wave) velocity (Castagna et al. 1985; Han et al. 1986; Prasad 2002; Zimmer 2003). Empirical relations established for consolidated and unconsolidated sediments to estimate S-wave velocity estimation 


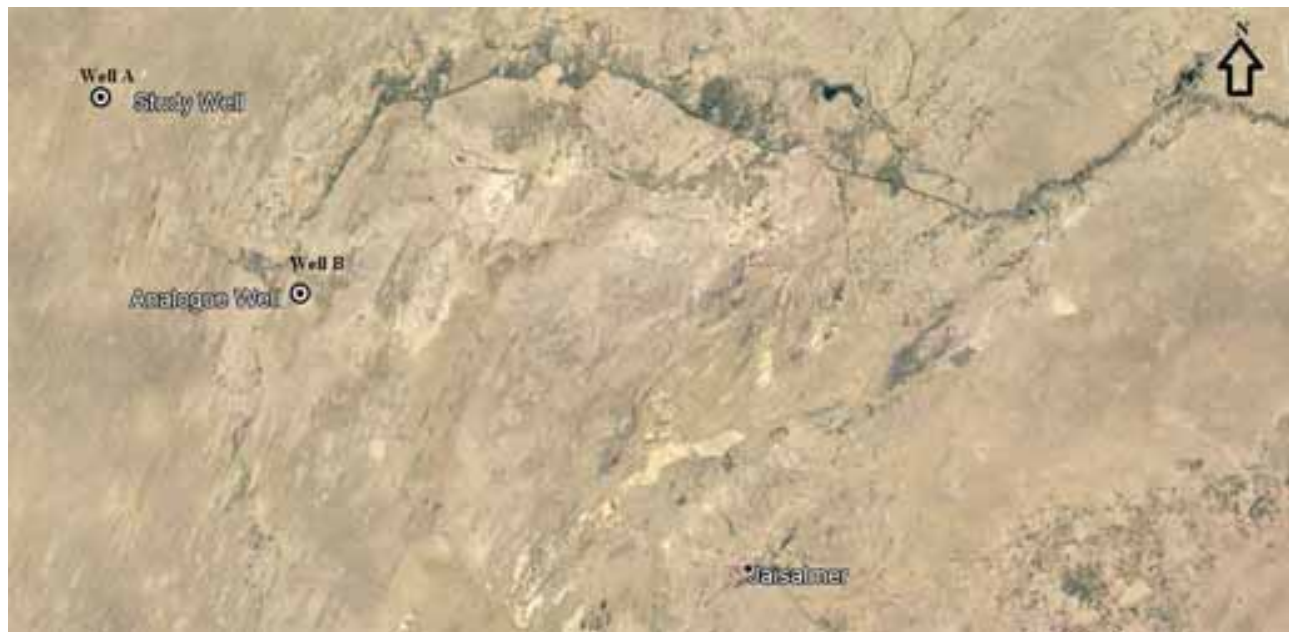

Figure 1. Location of study well A and analogue well B at Thar Desert in Rajasthan province near Jaisalmer city (modified after Sharma 2007).

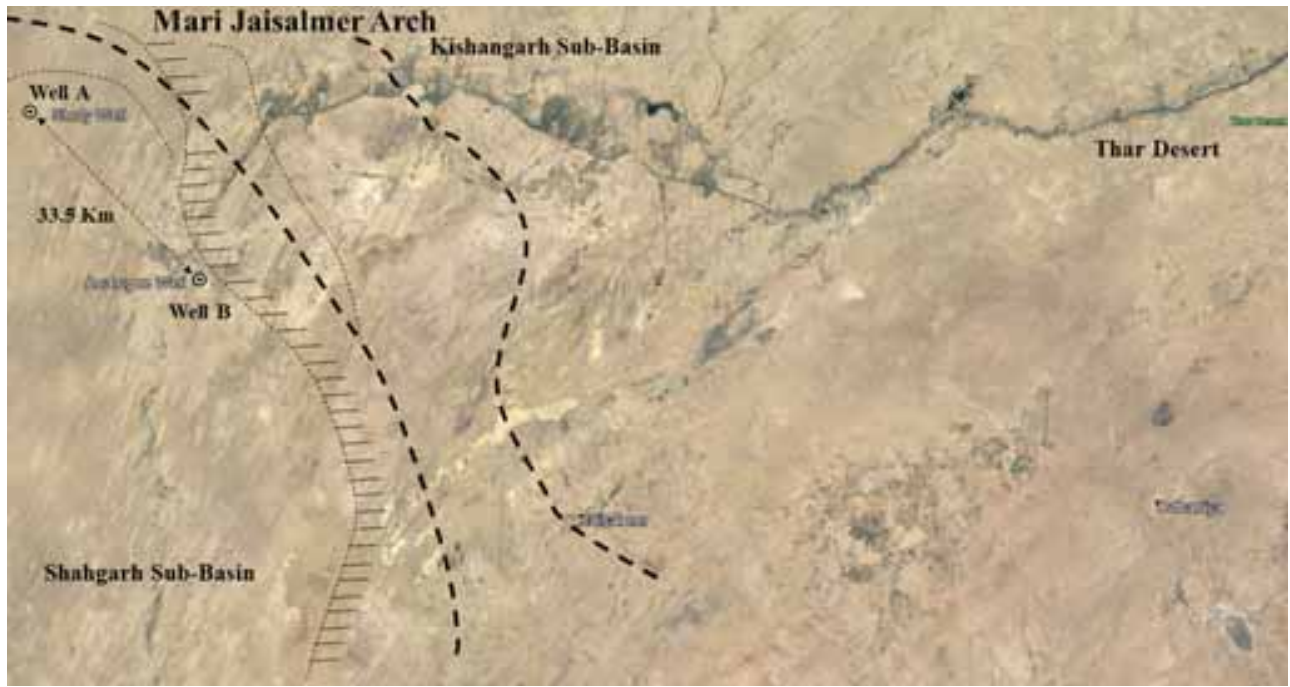

Figure 2. Tentative study area with faulted structures and study and analogue wells in Jaisalmer sub-basin, Rajasthan.

from P-wave (Han et al. 1986; Prasad and Nur 2003) for both P-wave velocity were more than and less than $1 \mathrm{~km} / \mathrm{s}$ (Lee 2010). We found more realistic $\mathrm{S}$-wave velocity estimation from $\mathrm{P}$-wave velocity data (more than $1 \mathrm{~km} / \mathrm{s}$ ) for this kind of lithology. The estimated shear sonic based on the experimental result was more feasible and accepted based on used analogue well log data response of the nearby area of the same sedimentary basinal structure support. We considered one component of S-wave velocity ( $X$-component) from the empirical relation based on the estimated result (Han et al. 1986; Prasad 2002; Lee 2010) whereas another one ( $Y$-component) evaluated based on the comparative analysis of analogue well data with the help of compressional and S-wave velocity changes. Our study well was drilled almost 33 yrs ago, mostly conventional well log data are available, and the interpretation approach was mostly conventional. The current study shows the essentiality towards the incorporation of anisotropy to get the exact reservoir target.

\section{Study area}

The Jaisalmer sub-basin encompasses an area of $30,000 \mathrm{~km}^{2}$ (figure 2) (Sharma 2007) situated in the western part of the country of the Rajasthan basin. It expands to the Mari region in the further western part of the country. Initially, the Rajasthan basin was fragmented from the Indus basin. Indus basin is one of the significant petroliferous basins on the global map.

Rajasthan basin comprises three sub-basins Jaisalmer, Bikaner-Nagaur and Barmer-Sanchor. 
The Indus basin was distributed into four significant structures - the Mari-Jaisalmer Arch, Shahgarh, Miajalar and the monoclinal Kishangarh.

Our study comes under the Jaisalmer sub-basin. This sub-basin evolved in the age of Mesozoic and Cenozoic. The major sedimentary units of this subbasin are Shumar, Bandah, Khuiala, Sanu, Parh, Upper Goru, Lower Goru and Pariwar formations. Figure 2 shows two wells A and B used for this study placed approximately $33.5 \mathrm{~km}$ apart from each other. These wells drilled to test and delineate the hydrocarbon potentiality in Mesozoic and Tertiary sequences. Pariwar and Goru formations are the primary formations of interest for hydrocarbon exploration. In 1986, well A was drilled, whereas well B was considered as the analogue support. Well A was drilled in the Ghotaru region to encounter the great Ghotaru structure whereas well B was drilled in the Bandha region in Jaisalmer sub-basin in the part of the large western Rajasthan shelf, west of the Aravalli mountains of Rajasthan province. The detailed study shows that well A was drilled in between the Kishangarh shelf and Shahgarh depression areas whereas well B was drilled at the high Kishangarh shelf. The study was performed with a focus on the Goru formation in both the wells, A and B.

\section{Methodology}

The vertically transverse isotropy (VTI) medium is explained effectively by five independent elastic stiffness coefficients. The vertical component of a transversely isotropic medium can also be demonstrated through three dimensionless anisotropy parameters such as epsilon $(\epsilon)$, gamma $(\gamma)$ and delta $(\delta)$ (Thomsen 1986). Initially, all these parameters come from generalised Hook's law which produces the stiffness factors tensor.

Generalised Hook's law is given by

$$
\sigma_{i j}=m_{i j k l} \epsilon_{k l}-\alpha P_{p} .
$$

The stiffness tensor can be written based on two keys as follows:

$$
m_{i l}=\left[\begin{array}{cccccc}
m_{11} & m_{12} & m_{13} & 0 & 0 & 0 \\
m_{21} & m_{22} & m_{23} & 0 & 0 & 0 \\
m_{31} & m_{32} & m_{33} & 0 & 0 & 0 \\
0 & 0 & 0 & m_{44} & 0 & 0 \\
0 & 0 & 0 & 0 & m_{55} & 0 \\
0 & 0 & 0 & 0 & 0 & m_{66}
\end{array}\right] .
$$

Propagation of borehole velocity comprises three components, such as $X, Y$ and $Z$ direction. The anisotropy factors is estimated, based on these directional changes. We considered three planes of the axis, $X_{1}, X_{2}$ and $X_{3}$ for the distribution of velocity.

Initially, we considered the vertical component as $X_{3}$ rotating under the equilibrium condition according to regular two index rotation of the other two axes (Dobson 1985; Nye 1985; Higgins et al. 2008). Under this condition, generalised Hook's law (equation 1) is applied over the velocity distribution. Significant estimated stiffness coefficients derived from the tensor matrix (equation 2) are presented in equation (3):

$$
\begin{aligned}
& m_{11}=m_{22}, m_{33}, m_{12}=m_{21}, \\
& m_{13}=m_{23}=m_{32}=m_{31}, \\
& m_{44}=m_{55}, m_{66}=\left(m_{11}-m_{12}\right) / 2 .
\end{aligned}
$$

We estimated the variation of the velocity direction, in the VTI medium with the help of Thomsen matrix distribution. The parameters used for this study are $\epsilon, \gamma$ and $\delta$ (Thomsen 1986) which are not three dimensional. The density remains unchanged throughout the body when the medium is isotropic. In the case of the isotropic medium, the values of the Thomsen anisotropy parameters, i.e., $\epsilon, \gamma, \delta$ will lead to zero, or else their value might act as the anisotropy intensity (Tsvankin 2005).

Shear sonic data were not available in the study well A. We used empirical relation (Han et al. 1986; Prasad 2002; Lee 2010) for estimating shear sonic data. The estimated S-wave velocity was considered as the $X$-component of the same whereas we calculated the $Y$-component of the S-wave velocity from relative analysis with analogue well $\mathrm{B}$ with the help of $\mathrm{P}$ - and S-wave data variation. The depth of investigation for well A restricted from 955 to $991 \mathrm{~m}$ in TVDSS (true vertical depth subsea - the actual depth of formation from mean sea level). We selected analogue well $\mathrm{B}$, based on similar geological conditions and litho-facies distribution of the nearby area. The depth of well $\mathrm{B}$ is restricted from 220 to $270 \mathrm{~m}$ in TVDSS (true vertical depth subsea - the actual depth of formation from mean sea level). We correlated the shale section of Goru formation regionally where we found the shale top of study well A at $955 \mathrm{~m}$ depth of the same formation identified with well B at 220 $\mathrm{m}$ of depth. The bottom part of this shale formation was identified at $991 \mathrm{~m}$ depth in well $\mathrm{A}$ and $270 \mathrm{~m}$ depth at well $\mathrm{B}$, respectively (figure 3 ). 


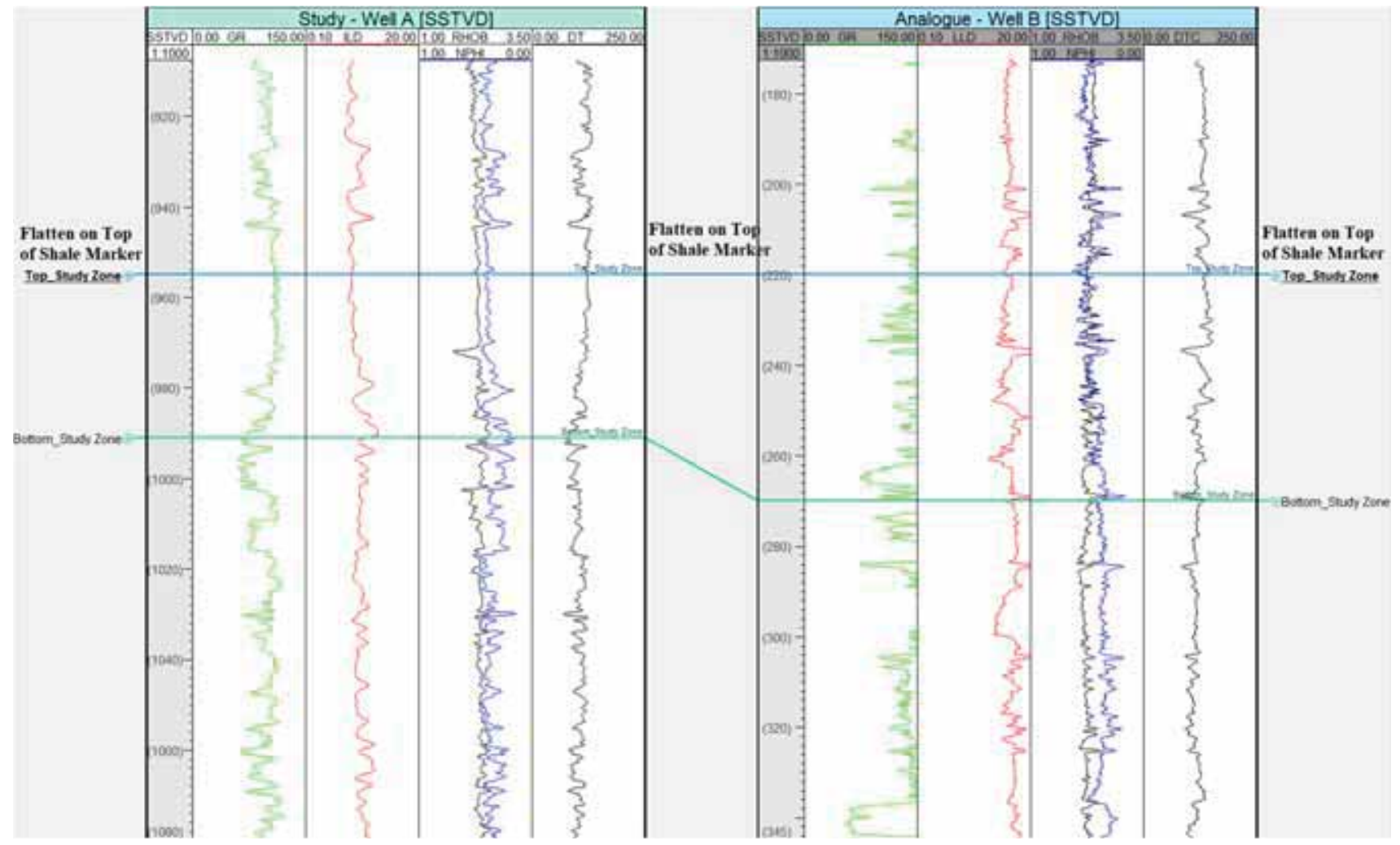

Figure 3. Well log correlation between study well A and analogue well B in the zone of investigation of Goru formation in Jaisalmer sub-basin.

A mild deviation in compressional sonic log was observed between well A and well B as a correlatable marker. Shear sonic data were not recorded in 1986 in the study well A. We took shear sonic data from well B, acquired in 2014. Both compressional and shear sonic data are essential for the anisotropy analysis.

We estimated S-wave velocity from the empirical relation of the sediments (consolidated and unconsolidated) where $\mathrm{P}$-wave velocity is more than 1 km/s (Han et al. 1986; Prasad 2002; Lee 2010):

$$
V_{\mathrm{p}}=2.286 V_{\mathrm{s}}^{0.672} .
$$

We did a critical review of both estimated result and nearby analogue well data for S-wave velocity. This estimated S-wave velocity of well A was considered as an $X$-component of S-wave velocity based on analogue assessment. We observed the deviation of compressional velocity in both the wells A and B. At the same time, we found two different evaluations ( $X$ - and $Y$-component) for field acquired $\mathrm{S}$-wave velocity in analogue well $\mathrm{B}$. These deviations acted as a critical role for estimating the $Y$-component of S-wave velocity of the study well A from well B.
The shear wave velocity was estimated from equation (4) with the help of compressional wave velocity considered as $X$-component of $\mathrm{S}$-wave velocity. We considered this component based on the qualitative analysis with $X$-component S-wave velocity of analogue well B. To get the $Y$-component of S-wave velocity we performed a qualitative analysis between study well $\mathrm{A}$ and analogue well B. Initially, we estimated the S-wave velocity changes for both components ( $X$ and $Y$ ). After that, we captured the compressional velocity changes between study well A and analogue well B to get the lithology variation. Finally, a product of these two variations was considered as the qualitative value to derive the $Y$-component of the $\mathrm{S}$-wave velocity in study well A. This approach we took for study well A since field acquired S-wave data were not available. Once both components of $\mathrm{S}$-wave velocity were estimated in well $\mathrm{A}$, Thomsen parameters were estimated for anisotropy analysis in the study well. Compressional and shear wave were recorded throughout the formation according to the depth of investigation in the study well. Figure 4 shows the variation of $\mathrm{P}$ - and S-sonic and density well $\log$ data in the zone of investigation. We found that the study well is a vertical well; 

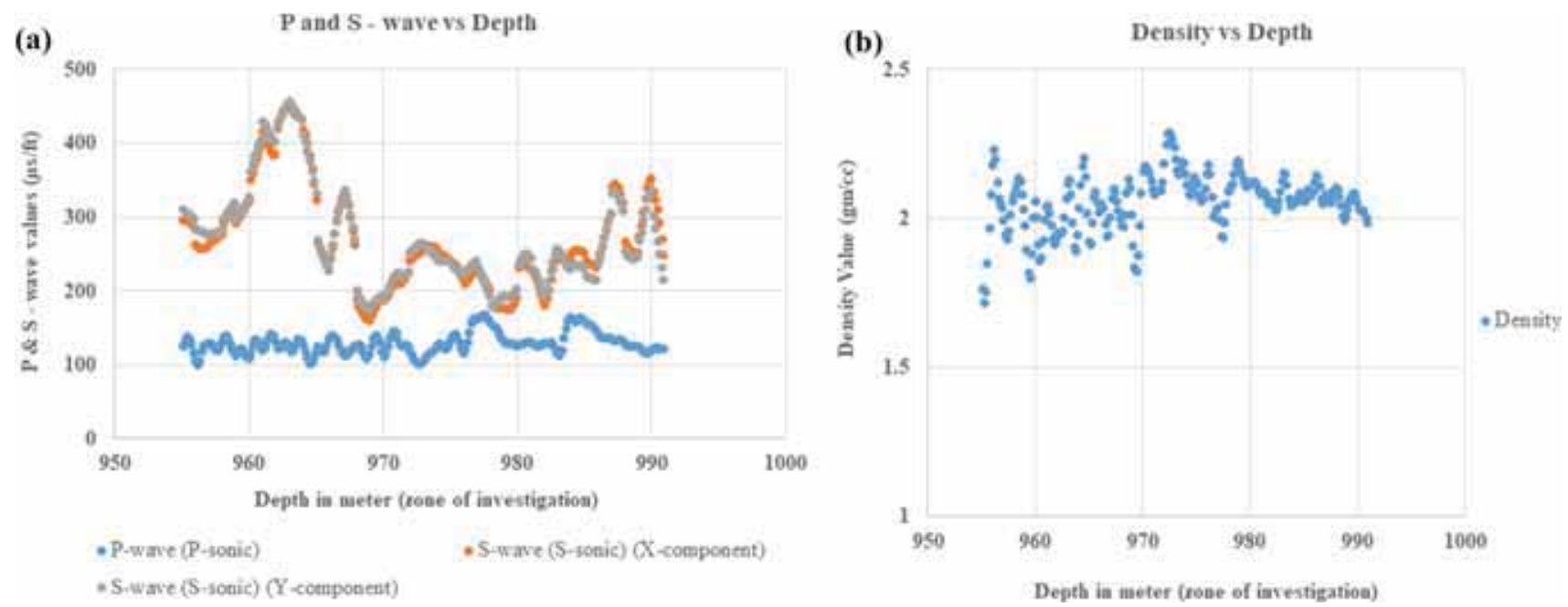

Figure 4. Capturing the variation of compressional sonic (P-wave), computed shear sonic (S-wave; $X$ - and $Y$-component) and density well $\log$ data with depth in the zone of investigation of the study well A.
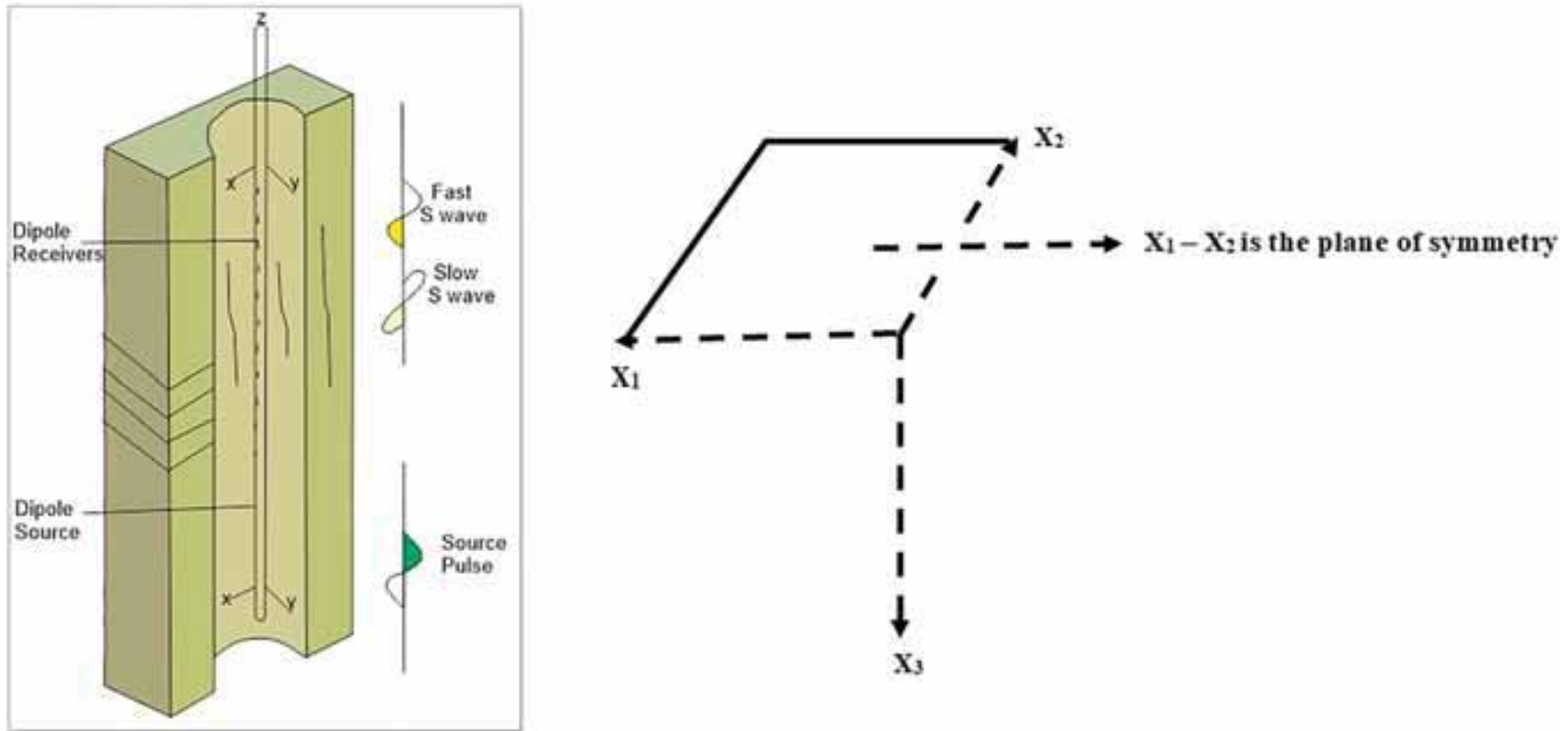

Figure 5. Schematic diagram for a qualitative demonstration for anisotropy calculation with respect to the plane of symmetry (modified after Ostadhassan et al. 2012).

hence $X_{3}$ was considered as the borehole axis. We used all assumptions for a vertical well. Hence, the formation will become isotropic in the $X_{1}-X_{2}$ planes (figure 5).

In a transverse isotropic medium, Thomsen anisotropy parameters illustrated through the propagation of compressional wave velocity directed as the vertical and shear wave velocity along the axis in the revolving condition $\left(X_{3}\right)$ and three dimensionless anisotropy parameters (Mavko et al. 2009) described as

$$
\epsilon=\left(m_{11}-m_{33}\right) / 2 m_{33},
$$

$$
\begin{gathered}
\gamma=\left(m_{66}-m_{55}\right) / 2 m_{55}, \\
\delta=\left(\left(m_{13}+m_{55}\right)^{2}-\left(m_{33}-m_{55}\right)^{2}\right) \\
/ 2 m_{33}\left(m_{33}-m_{55}\right) .
\end{gathered}
$$

The expression for $\delta$ is a more complex quantity. Out of all the three parameters, $\delta$ becomes the most important parameter as it explains the NMO behaviour of VTI layers (Tsvankin 1997).

Let the velocity of a wave travelling in the axis of $X_{i}$ and polarised along the axis of $X_{j}$ be $V_{i j}$. The 
relation between $V_{i j}$ and $m_{i j}$, in the case of a transverse isotropic case is (Ostadhassan et al. 2012)

$$
\begin{aligned}
& V_{11}=V_{22}=\sqrt{\left(\frac{m_{11}}{\rho}\right)}, V_{33}=\sqrt{\left(\frac{m_{33}}{\rho}\right)}, \\
& V_{12}=V_{21}=\sqrt{\left(\frac{m_{66}}{\rho}\right)}, \\
& V_{13}=V_{31}=V_{23}=V_{32}=\sqrt{\left(\frac{m_{55}}{\rho}\right)} .
\end{aligned}
$$

For the entire characterisation of a VTI medium, $m_{33}, m_{55}, m_{66}, m_{11}$ and $m_{13}$ are required. With the help of equation (8), stiffness coefficients $m_{33}, m_{55}$, $m_{66}$ were calculated and $m_{11}$ and $m_{13}$ were estimated using ANNIE model assumptions. The ANNIE model (Shoenberg et al. 1996) is a widely used anisotropic model. This model obtains the two coefficients by considering two assumptions. We obtained the first assumption as a consequence of the seismic observations that the velocity of NMO is small compared to vertical velocity; hence, Thomsen parameter $\delta$ can be set to zero (equation 7 ) i.e.,

$$
m_{13}+2 m_{44}-m_{33}=0 .
$$

In the above expression, for a VTI medium, we $m_{44}$ and $m_{55}$ were considered as a variable parameter to replicate each term.

We observed that in many cases $m_{13}=m_{12}$ for shale formation; this equality approach was considered as the second assumption, i.e.,

$$
\begin{aligned}
& m_{13}=m_{12}=m_{11}-2 m_{66}, \\
& m_{66}-m_{44}=\left(m_{11}-m_{33}\right) / 2 .
\end{aligned}
$$

From this it follows that

$$
\left(m_{11}, m_{13}\right)=f\left(m_{33}, m_{44}, m_{66}\right) .
$$

After substituting all the five stiffness constants $\left(m_{11}, m_{13}, m_{33}, m_{55}, m_{66}\right)$ in equations (5-7), we obtained the values of $\epsilon, \gamma$ and $\delta$. Two major factors for the estimation of Thomsen parameters are $V_{12}$ and $V_{13}$. We considered the $Y$-component of the $\mathrm{S}$-wave velocity as $V_{12}$ whereas the $X$-component was considered as $V_{13}$ of the study well A. The constant $\epsilon$ again defined as the fractional difference among the $\mathrm{P}$-wave velocity in the two directions, i.e., in the vertical $\left(V_{\mathrm{P}}\left(90^{\circ}\right)\right)$ and horizontal $\left(V_{\mathrm{P}}\left(0^{\circ}\right)\right)$ showing P-wave anisotropy (Ruger 1998; Simm and Bacon 2014), i.e.,

$$
\epsilon=\left(V_{\mathrm{P}}\left(90^{\circ}\right)-V_{\mathrm{P}}\left(0^{\circ}\right)\right) / V_{\mathrm{P}}\left(0^{\circ}\right) .
$$

Similarly, the constant $\gamma$ describes the same characteristics for S-wave which is equal to the difference between the vertical $\left(V_{\mathrm{SH}}\left(90^{\circ}\right)\right)$ and horizontal propagations $\left(V_{\mathrm{SH}}\left(0^{\circ}\right)\right)$ of the horizontally propagating S-wave (Simm and Bacon 2014; Avseth 2015), i.e.,

$$
\gamma=\left(V_{\mathrm{SH}}\left(90^{\circ}\right)-V_{\mathrm{SH}}\left(0^{\circ}\right)\right) / V_{\mathrm{SH}}\left(0^{\circ}\right) .
$$

The variation of calculated stiffness factors and Thomsen parameters with depth is presented in figure 6 . Then the incorporation of $\epsilon$ and $\gamma$ values to the compressional and shear wave data using the above equation (equations 12 and 13).

We captured the lithology variation in shale dominated clay and limestone mixed section based on the comparative study between $\mathrm{P}$ - and S-wave velocity ratio and P-impedance as well as Poisson's ratio and $\mathrm{P}$-impedance. We obtained the support between the time processing parameter of the compressional wave $(\eta)$ and Thomsen parameter $\delta$. We estimated $\eta$ from the following expression (equation 14) (Alkhalifah and Tsvankin 1995):

$$
\eta=(\epsilon-\delta) /(1+2 \delta) \text {. }
$$

\section{Results and discussion}

We performed a comparative study between conventional compressional and shear velocity with anisotropy incorporated compressional and shear velocity study through cross plot analysis.

Figure 7 represents the cross-plot of P-wave velocity vs. depth and the effect of anisotropy on the P-wave velocity. We observed few significant changes in the trend of compressional velocity in the zone of investigation in the study well. We got the maximum compressional velocity of the study well A, at a depth of $956 \mathrm{~m}$ which is around $3054 \mathrm{~m} / \mathrm{s}$ whereas we observed around $3000 \mathrm{~m} / \mathrm{s}$ velocity at a depth of 965 and $973 \mathrm{~m}$. However, after the incorporation of the anisotropy factor $\epsilon$, the maximum velocity increases, and we observed that at a depth of $956,965,967$ and $973 \mathrm{~m}$, it is around $3100 \mathrm{~m} / \mathrm{s}$. Similarly, without the effect of the anisotropy factor minimum, $\mathrm{P}$-wave velocity is around $1812 \mathrm{~m} / \mathrm{s}$ at a depth of $977 \mathrm{~m}$; however, with the effect of $\epsilon$ it reduces to $1630 \mathrm{~m} / \mathrm{s}$ in the same study well A at the same depth. Anisotropyincorporated velocity looks more practical based on the sub-surface geological nature and variability. The standard P-wave velocity was estimated directly from $\mathrm{P}$-sonic data where noises were present and directional changes were observed in the 

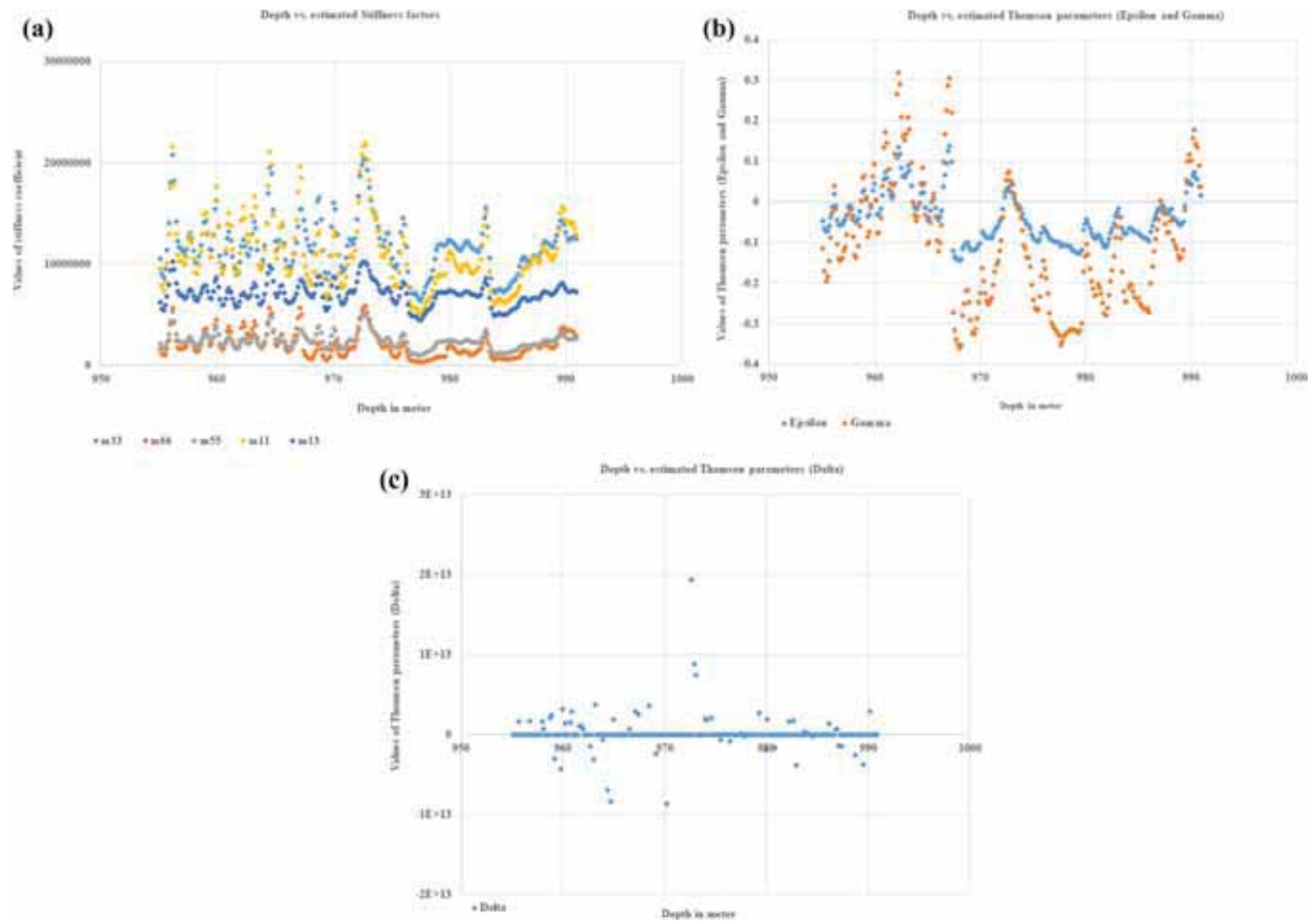

Figure 6. Capturing the computed (a) stiffness coefficients $\left(m_{11}, m_{13}, m_{33}, m_{55}, m_{66}\right)$ with depth; (b) Thomsen parameters $(\epsilon$ and $\gamma$ ) with depth and (c) Thomsen parameter $\delta$ with depth in the zone of investigation of the study well A.

P-wave or compressional wave velocity. Alternation of lithology was not captured adequately based on P-wave velocity and the related study (Datta Gupta et al. 2012). The trend of P-wave velocity changed after the incorporation of anisotropy parameters where distinct changes occurred at 967, 977 and $984 \mathrm{~m}$ depth. The incorporation of Thomsen parameters makes $\mathrm{P}$-wave velocity more reliable, and a small variation of lithology was captured based on anisotropy incorporated P-wave velocity analysis and its related study (Datta Gupta and Gupta 2017). We found $-13.38 \%$ to $14.25 \%$ variation of $\mathrm{P}$-wave velocity after the incorporation of the anisotropy factor where Pwave anisotropy $(\epsilon)$ we found approximately $5.99 \%$ based on an estimation of standard deviation.

Figure 8 illustrates the cross-plot of the shear or S-wave velocity $v s$. depth or general trend and anisotropy incorporated trend. We noticed that some differences occur between normal and anisotropy incorporated trend for the $X$ - and $Y$ component of the S-wave velocity in the study well A. In both the cases for the $X$ - and $Y$-component, the S-wave velocity shows significant variations up to a depth of $967 \mathrm{~m}$ and then sudden changes observed in the velocity occurred at a depth of $976 \mathrm{~m}$. Another sudden change in velocity took place at a depth of $984 \mathrm{~m}$. In a typical case maximum S-wave velocity for the $X$-component, we observed at a depth of $956 \mathrm{~m}$ which is around 1539 $\mathrm{m} / \mathrm{s}$ whereas for the $Y$-component we found it at $967 \mathrm{~m}$ depth with the value of around $1650 \mathrm{~m} / \mathrm{s}$. After the incorporation of the anisotropy factor $\gamma$, it increases for $X$ - and $Y$-component of S-wave velocity and shows values of around 1697 and 2152 $\mathrm{m} / \mathrm{s}$ at a depth of $967 \mathrm{~m}$.

Similarly, in the general case, at a depth of 977 and $978 \mathrm{~m}$, we got minimum S-wave velocity for $X$ - and $Y$-component which is around 708 and $389 \mathrm{~m} / \mathrm{s}$. However, at the same depth after the incorporation of the anisotropy factor $\gamma$, it decreases to around 464 and $252 \mathrm{~m} / \mathrm{s}$ at a depth of $978 \mathrm{~m}$ for both the components of the S-wave velocity. The changes of the velocity gradient become smooth after the incorporation of the anisotropy factor. This observation supports to build a robust 
(a)

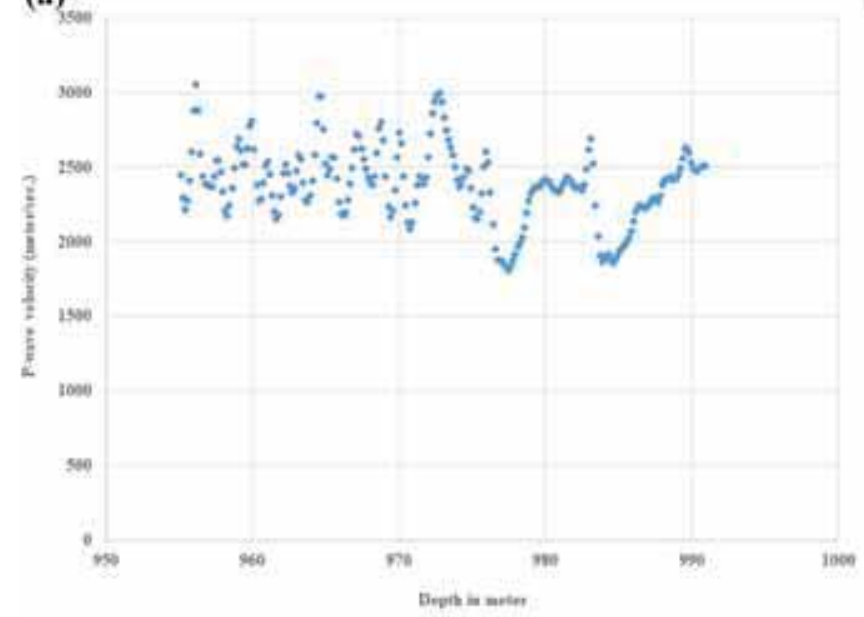

(b)

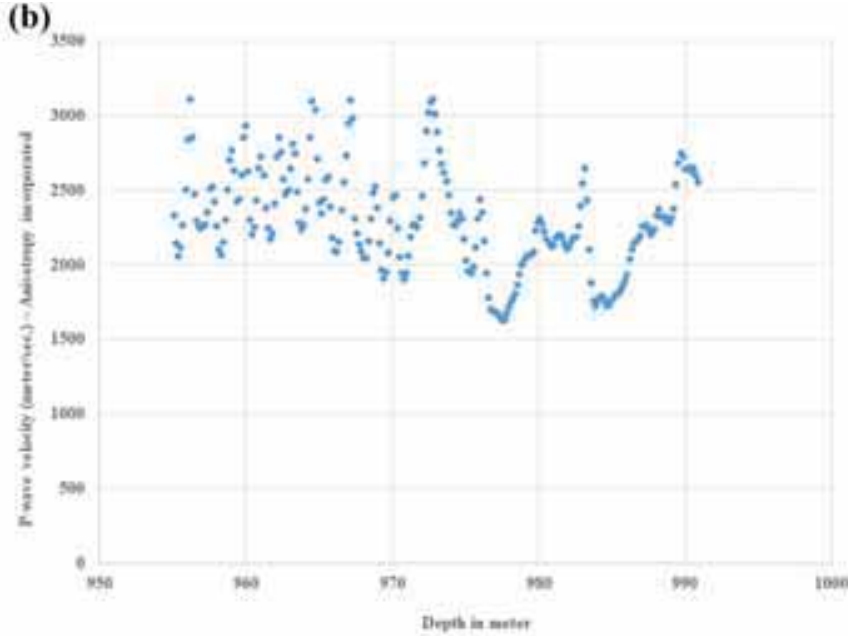

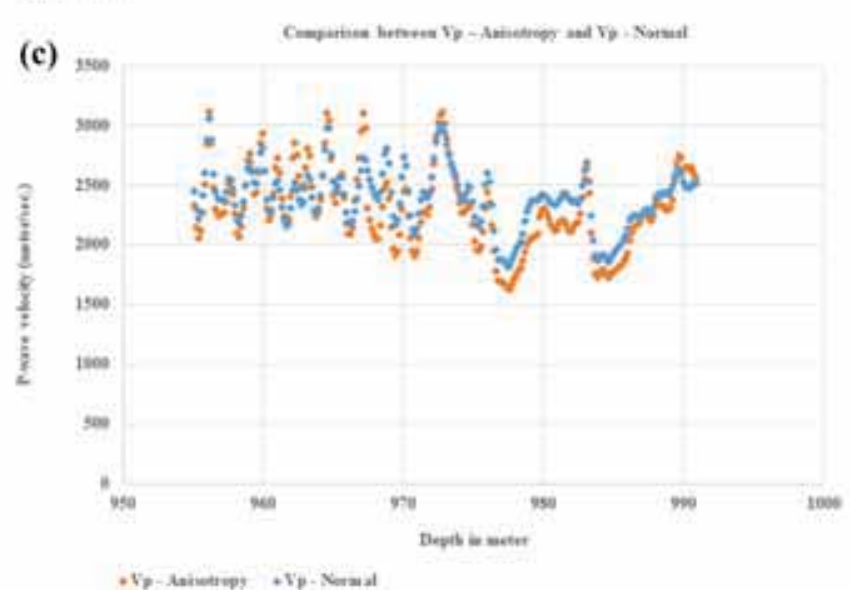

Figure 7. A comparison between standard (a) P-wave velocity derived conventionally; (b) anisotropy parameters incorporated $\mathrm{P}$-wave velocity; (c) composite changes of $\mathrm{P}$-wave velocity for both with and without the anisotropy incorporated factor.

multi-layered velocity model with the incorporation of the anisotropy factor. We observed that the standard velocity trend is comparatively noisy whereas the anisotropy incorporated one is less noisy and properly distinguished in different lithology. Similar to the P-wave velocity we found $-31.80 \%$ to $36.10 \%$ variation of S-wave velocity after the incorporation of the anisotropy factor where the S-wave anisotropy $(\gamma)$ we found was approximately $15.44 \%$ based on an estimation of standard deviation.

Figure 9 presents the cross plot between the estimated Thomsen parameters $\gamma$ and $\epsilon$ which are represented as $\mathrm{P}$-wave anisotropy $(\epsilon)$ and $\mathrm{S}$-wave anisotropy $(\gamma)$. We observed fair lithology (shale with clay and limestone) discrimination in anisotropy-incorporated velocities, where the shale section with clay dominated with mixes of limestone trend in the Goru formation. We found a good correlation between $\epsilon$ and the $\gamma$ plot with an $R^{2}$ value of 0.9838 . We found one trend line in this plot which follows the relationship between $\epsilon$ and $\gamma$ for this kind of lithology and is represented by equation (15) to establish lithology differentiations (Okorie et al. 2016):

$$
y=2.5557 x-0.0068
$$

where $y$ represents the $\mathrm{P}$-wave anisotropy $(\epsilon)$ and $x$ represents the S-wave anisotropy $(\gamma)$.

The lithology of the study section is dominated by shale and mixed with limestone and clay this scenario is presented in figure 9 . We found two separate trend lines for shale and petite clay dominated section with an $R^{2}$ value of 0.9944 and a limestone section $R^{2}$ value of 0.9821 . These lithological variations are represented by the following expressions:

$$
\begin{gathered}
y=2.5577 x-0.0067 \text { (limestone), } \\
y=2.4399 x-0.004 \text { (shale with little clay), }
\end{gathered}
$$

where $y$ represents the $\mathrm{P}$-wave anisotropy $(\epsilon)$ and $x$ represents the S-wave anisotropy $(\gamma)$. 

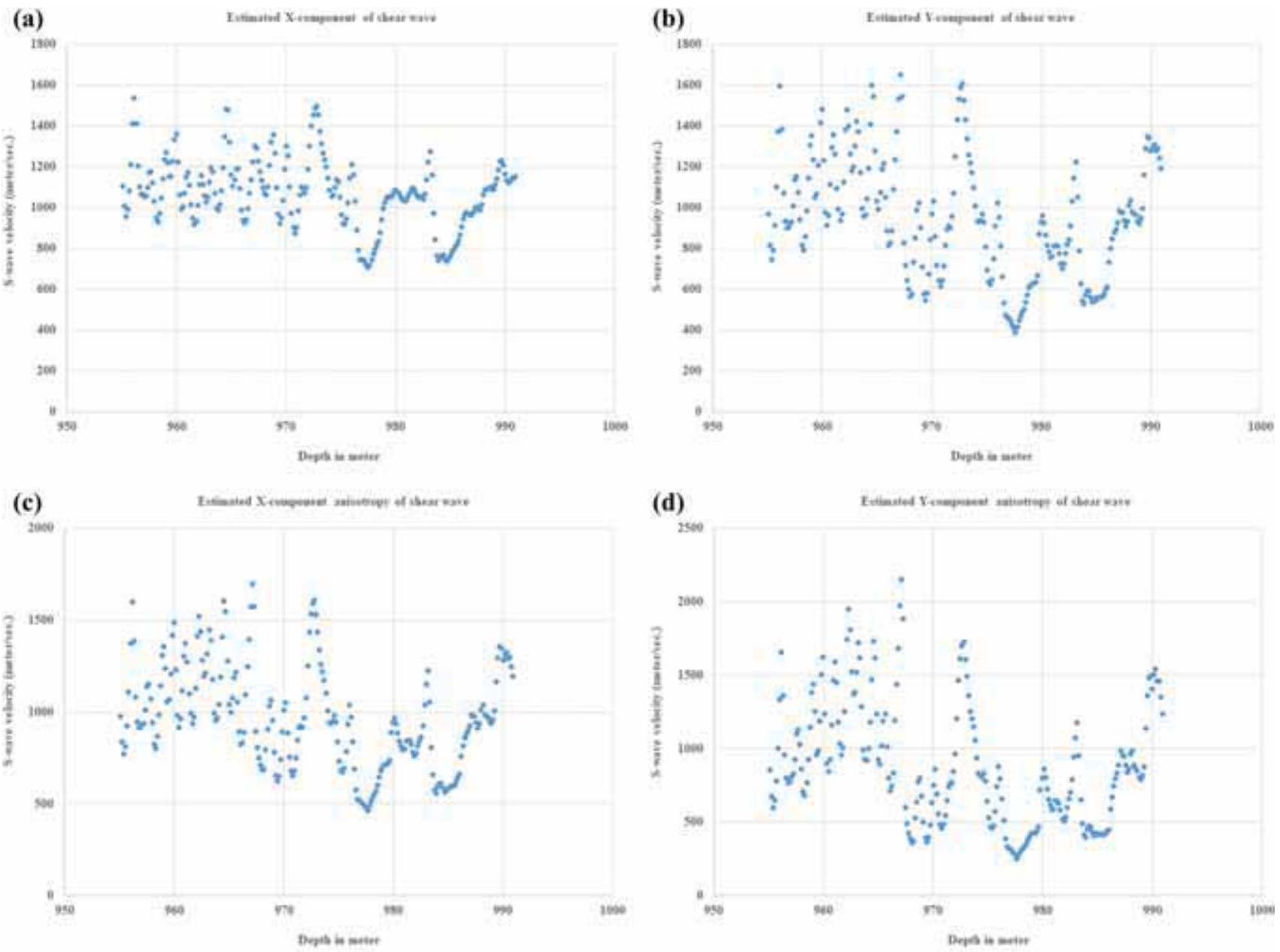

(e)

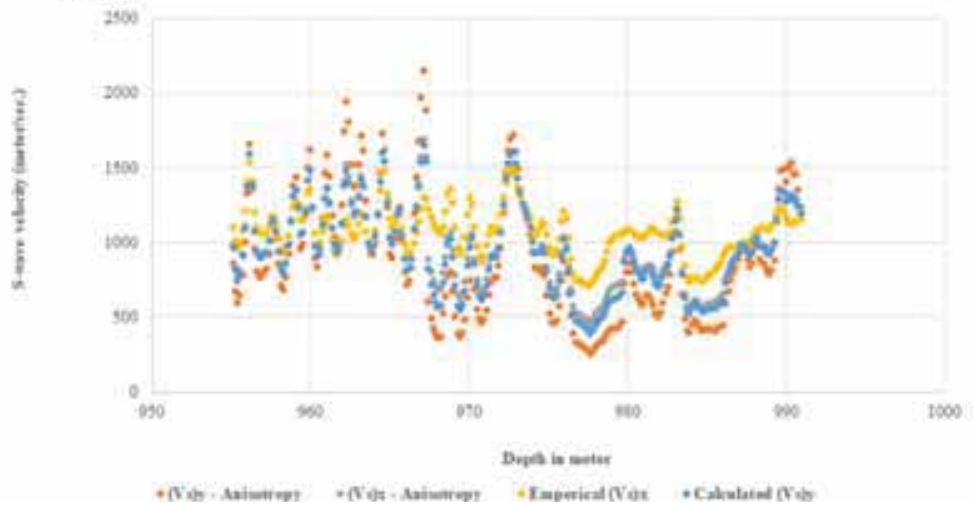

Figure 8. A comparison between standard S-wave velocities for both $X$ - and $Y$-component derived conventionally and anisotropy parameters incorporated S-wave velocity: (a) estimated $X$-component of the shear wave; (b) estimated $Y$-component of the shear wave; $(\mathbf{c})$ estimated $X$-component anisotropy of the shear wave; $(\mathbf{d})$ estimated $Y$-component anisotropy of the shear wave; (e) comparison between $V_{\mathrm{s}}$ - anisotropy and $V_{\mathrm{s}}$ - normal for both $X$ - and $Y$-component of the shear wave.

This lithology discrimination is a challenging job as shale and limestone are in the unsorted condition in the Goru formation, and the density/ $\mathrm{P}$-wave velocity gradient is too adjacent for both type of rock character in this formation. Figure 10 shows the velocity ratio of $\mathrm{P}$ - and $\mathrm{S}$-wave for both $X$ - and $Y$-component of the S-wave and acoustic impedance are not fruitful in separating these unsorted lithologies by the conventional approach. This separation will be essential for a further quantitative interpretation of sedimentary model development. P-impedance and $V_{\mathrm{p}} / V_{\mathrm{s}}$ assessment study are significant indicators of hydrocarbon fluid typing and hydrocarbon-bearing lithofacies 

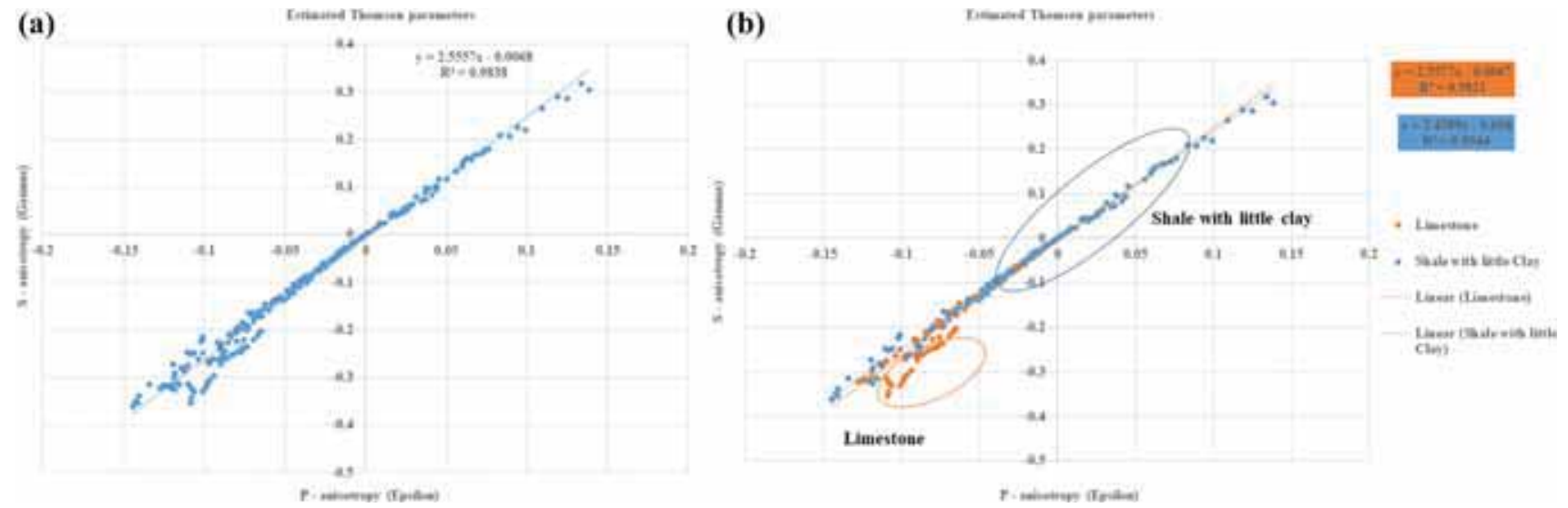

Figure 9. Cross plot between two Thomsen parameters of $\gamma$ and $\epsilon$ for the discrimination of lithology - shale with little clay and limestone lithology with the correlation between $\epsilon$ and $\gamma$ in identified lithology: (a) estimated Thomsen parameters plot between $\epsilon$ and $\gamma$ and (b) estimated Thomsen parameters plot between $\epsilon$ and $\gamma$ with identified lithology.
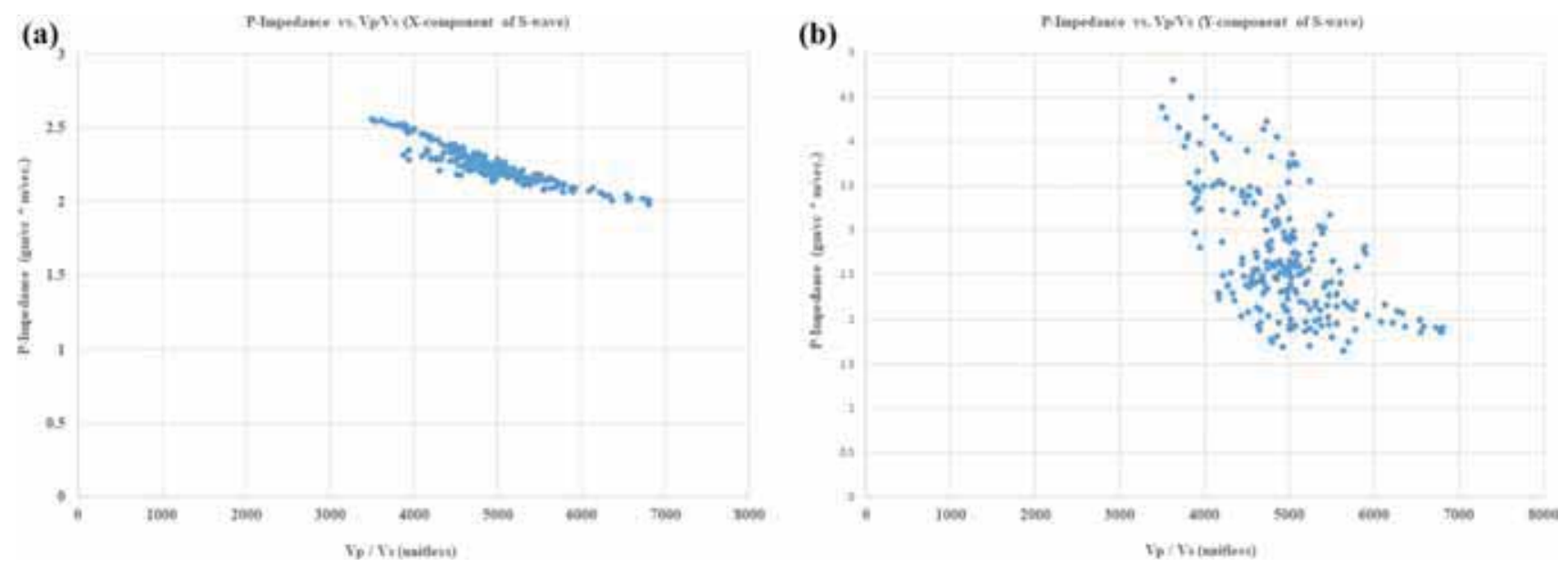

Figure 10. A comparison study between the conventionally derived velocity ratio of the $\mathrm{P}-$ and $\mathrm{S}$-wave $\left(V_{\mathrm{p}} / V_{\mathrm{s}}\right)$ and $\mathrm{P}$-impedance for both $X$ - and $Y$-component of S-wave velocity; the study shows the difficulties in the discrimination of unsorted lithology: (a) P-impedance vs. $V_{\mathrm{p}} / V_{\mathrm{s}}$ (X-component of the $\mathrm{S}$-wave) and (b) P-impedance vs. $V_{\mathrm{p}} / V_{\mathrm{s}}(Y$-component of the S-wave).
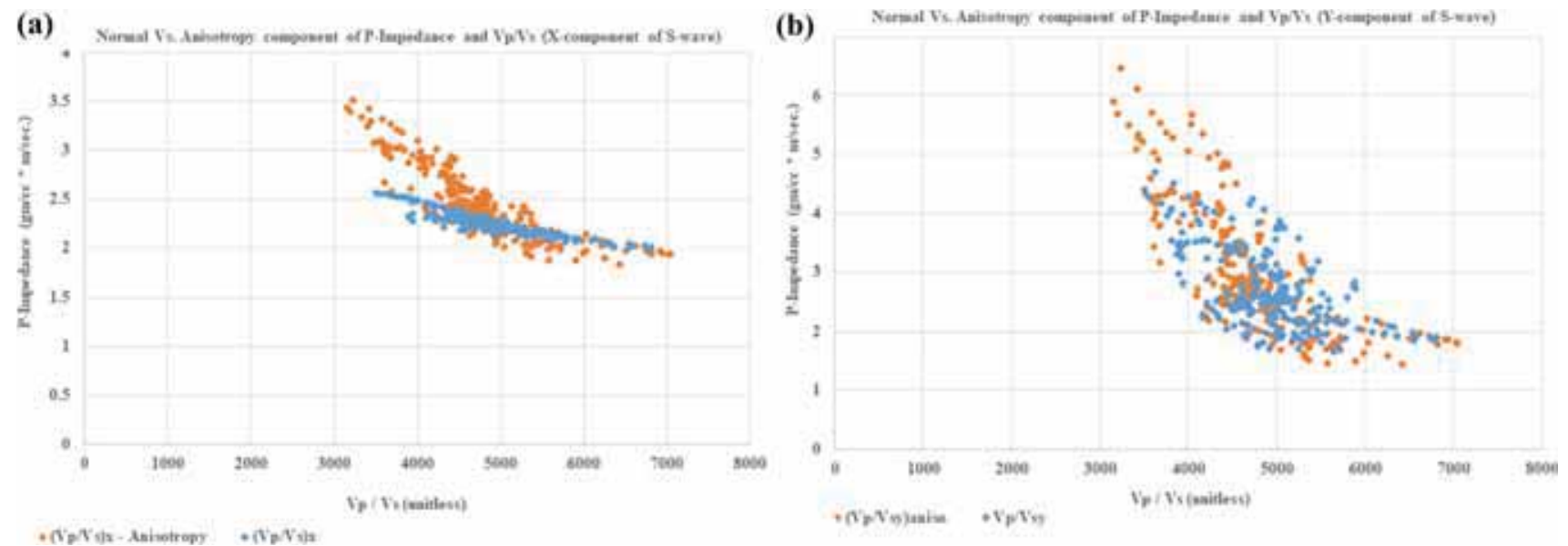

Figure 11. A comparison study between the velocity ratio of the $\mathrm{P}$ - and $\mathrm{S}$-wave $\left(V_{\mathrm{p}} / V_{\mathrm{s}}\right)$ (for the $X$ - and $Y$-component of the S-wave) with P-impedance derived from the conventional method and the anisotropy parameters incorporated method; significant changes observed in between conventional and anisotropy parameters incorporated result: (a) normal vs. anisotropy component of P-impedance and $V_{\mathrm{p}} / V_{\mathrm{s}}(X$-component of the S-wave) and (b) normal vs. anisotropy component of P-impedance and $V_{\mathrm{p}} / V_{\mathrm{s}}(Y$-component of the $\mathrm{S}$-wave). 

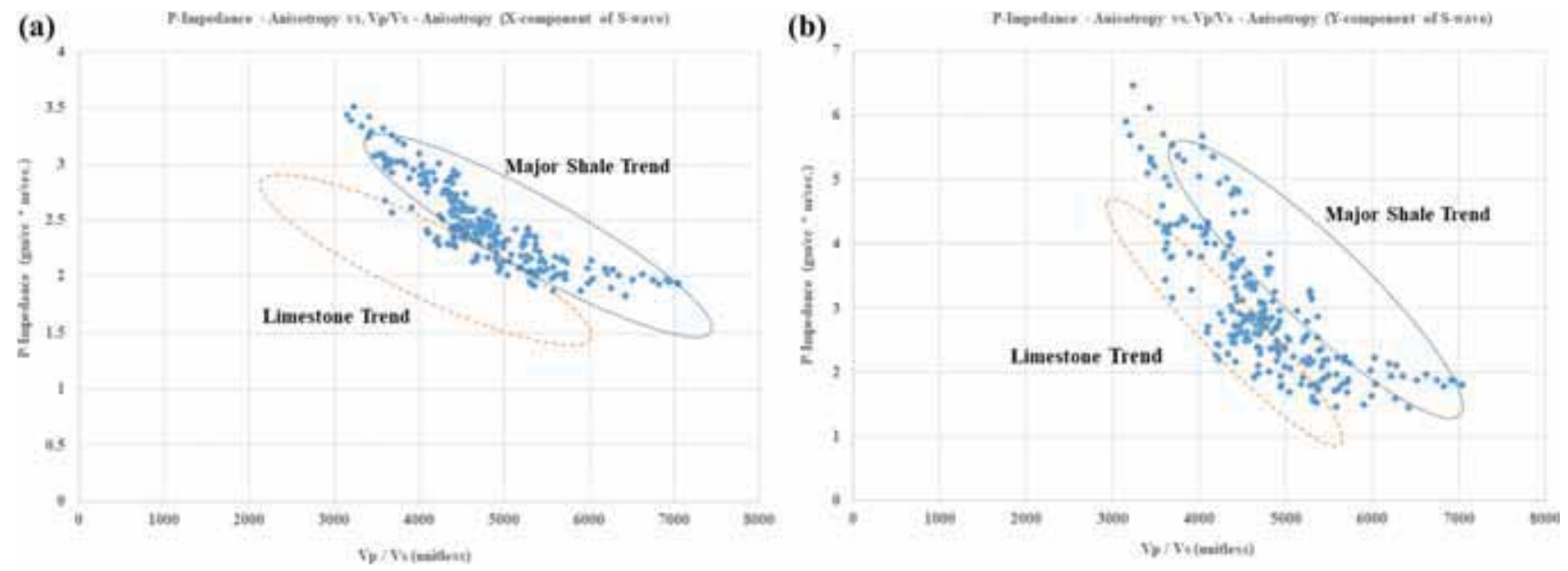

Figure 12. A comparison study between the anisotropy-incorporated velocity ratio of the $\mathrm{P}-$ and $\mathrm{S}-\mathrm{wave}\left(V_{\mathrm{p}} / V_{\mathrm{s}}\right)($ for both $X$ - and $Y$-component of the $\mathrm{S}$-wave velocity) and $\mathrm{P}$-impedance shows lithology discrimination between a major shale trend and a limestone trend: (a) P-impedance - anisotropy vs. $V_{\mathrm{p}} / V_{\mathrm{s}}$ - anisotropy (X-component of the S-wave) and (b) P-impedance anisotropy vs. $V_{\mathrm{p}} / V_{\mathrm{s}}$ - anisotropy ( $Y$-component of the S-wave).
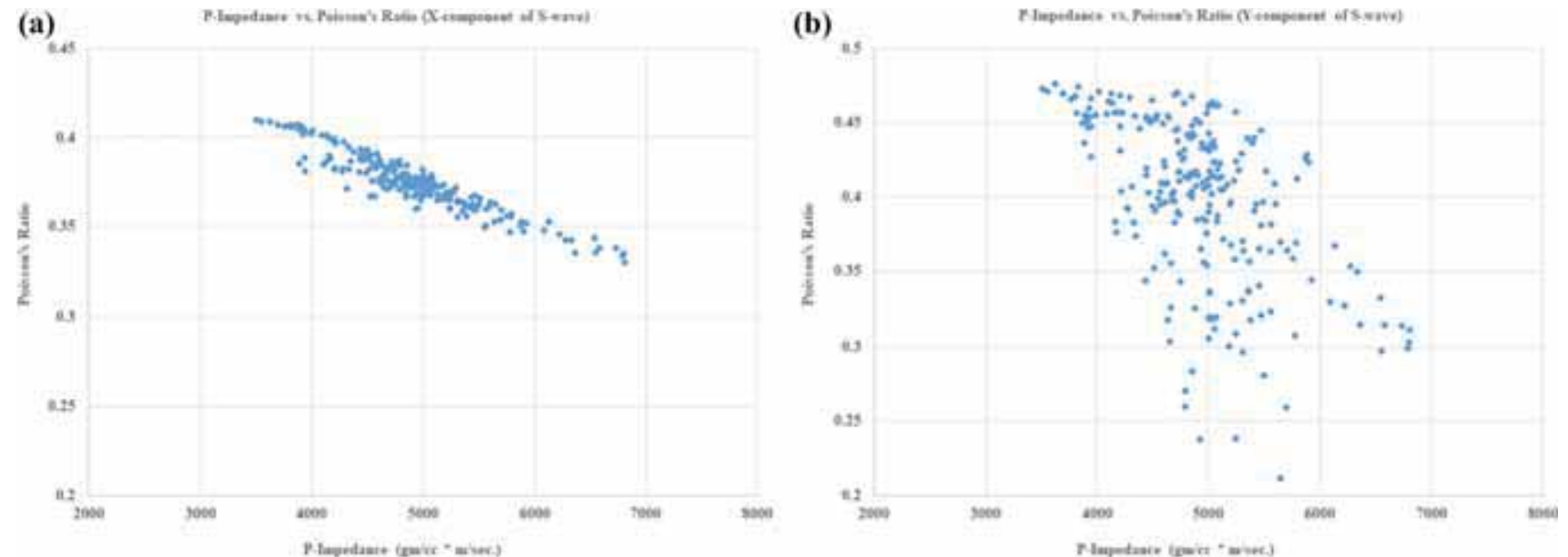

Figure 13. A comparative study between conventionally derived Poisson's ratio and P-impedance for both $X$ - and $Y$-component of S-wave velocity; the study shows the difficulties in the discrimination of unsorted lithology: (a) P-impedance vs. Poisson's ratio ( $X$-component of the S-wave) and (b) P-impedance vs. Poisson's ratio ( $Y$-component of the S-wave).

identification. In figure 11, we observed that significant deflection between anisotropy incorporated and without anisotropy incorporated result for P-impedance and $V_{\mathrm{p}} / V_{\mathrm{s}}$ result (including $X$ and $Y$-component of $\mathrm{S}$-wave velocity). This study leads to producing substantial changes in a comparative study between P-impedance and $V_{\mathrm{p}} / V_{\mathrm{s}}$ analysis during the inclusion of anisotropy factors in both variables (figure 12) which allows separating two major lithology trends - shale and limestone in this kind of unsorted lithology formation.

Another rock physics template study for lithology identification is the comparative analysis between the Poisson ratio and P-impedance. Initially, we faced difficulties in the separation of this lithology for the typical case (figure 13). We observed significant changes in between anisotropy incorporated and without anisotropy incorporated Poisson ratio and P-impedance values (figure 14). Figure 15 shows that anisotropy as a factor is essential for sub-surface geological interpretation, and as a result, anisotropy incorporated Poisson ratio and $\mathrm{P}$-impedance are capable of two separate dominant trends of lithology in the study formation - Goru. After elimination of a few outlier values, the comparative study between $\eta$ and $\delta$ supported the earlier observation for lithology identification with an $R^{2}$ value of 0.9833 and supported by equation (18) (figure 16). We found that the anisotropy of $\eta$ is $5.91 \%$ :

$$
y=0.3832 x+0.0017,
$$



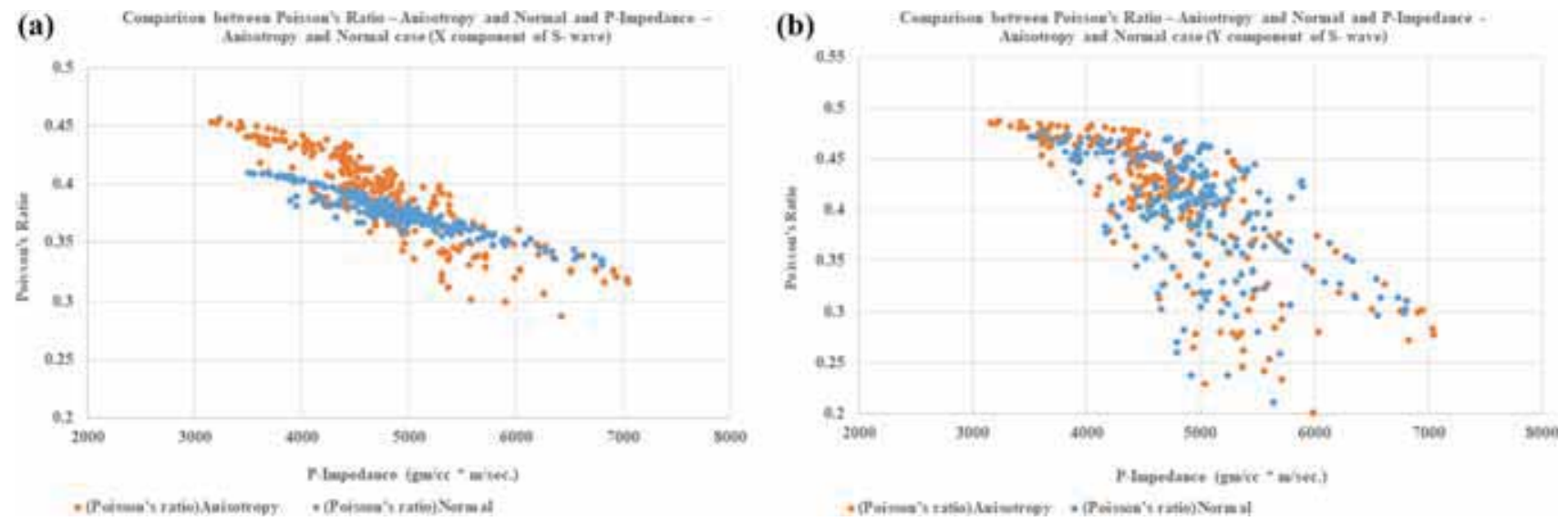

Figure 14. A comparison study between conventionally derived Poisson's ratio (for $X$ - and $Y$-component of S-wave velocity) with P-impedance derived from the conventional method and anisotropy parameters incorporated method; significant changes observed in between conventional and anisotropy parameters incorporated result: (a) comparison between Poisson's ratio anisotropy and normal and P-impedance - anisotropy and normal case ( $X$-component of the S-wave) and (b) comparison between Poisson's ratio - anisotropy and normal and P-impedance - anisotropy and normal case ( $Y$-component of the S-wave).
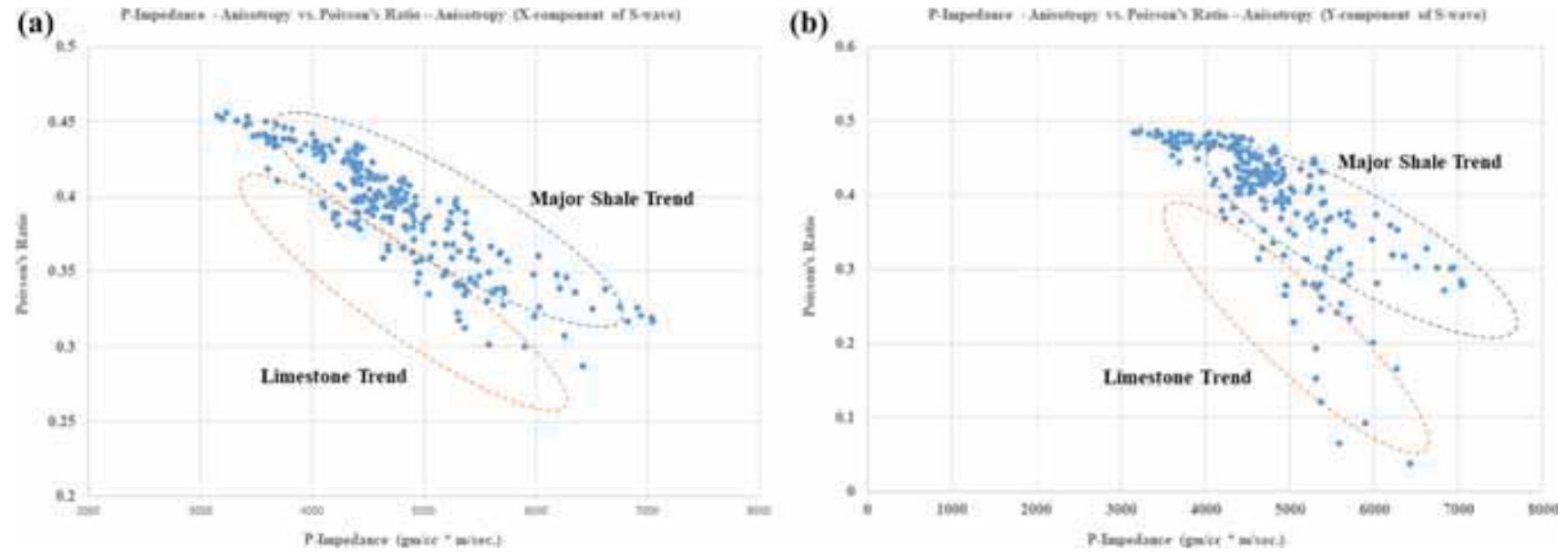

Figure 15. A comparative study between anisotropy incorporated Poisson's ratio (for $X$ - and $Y$-component of S-wave velocity) and P-impedance shows lithology discrimination between a major shale trend and a limestone trend: (a) P-impedance anisotropy vs. Poisson's ratio - anisotropy ( $X$-component of the S-wave) and (b) P-impedance - anisotropy vs. Poisson's ratio anisotropy ( $Y$-component of the S-wave).

where $y$ represents the Thomsen parameter $(\delta)$ and $x$ represents the $\mathrm{P}$-wave time processing parameter $(\eta)$.

We performed the current study on Goru formation in Ghotaru area of Jaisalmer sub-basin where the formation comprises mostly shale section but apart from shale, few thin sections of clay, silty clay and limestone are identified in this formation. These observations were confirmed from a drilled sample analysis of the study well A. Our present study was carried out in a tectonically faulted Ghotaru region for the measurement of anisotropy parameters which helps in lithology discrimination from unsorted rock character. The incorporation of Thomsen parameters in compressional and shear wave velocity demonstrates the heterogeneity of shale formation which is not available in conventional compressional and shear sonic data. The study was conducted with an old and limited number of well data set. Shear wave velocity data estimated from compressional velocity data using empirical relation and S-wave velocity of analogue well B was used for a further comparative study and estimation in the correlated depth of the study zone and used in well A for further anisotropy analysis. These changes will impact the full layered stacked 3D velocity model and quantitative interpretation for hydrocarbon exploration. The study area shows both positive and negative values of $\epsilon$ and $\gamma$ with a good correlation between 


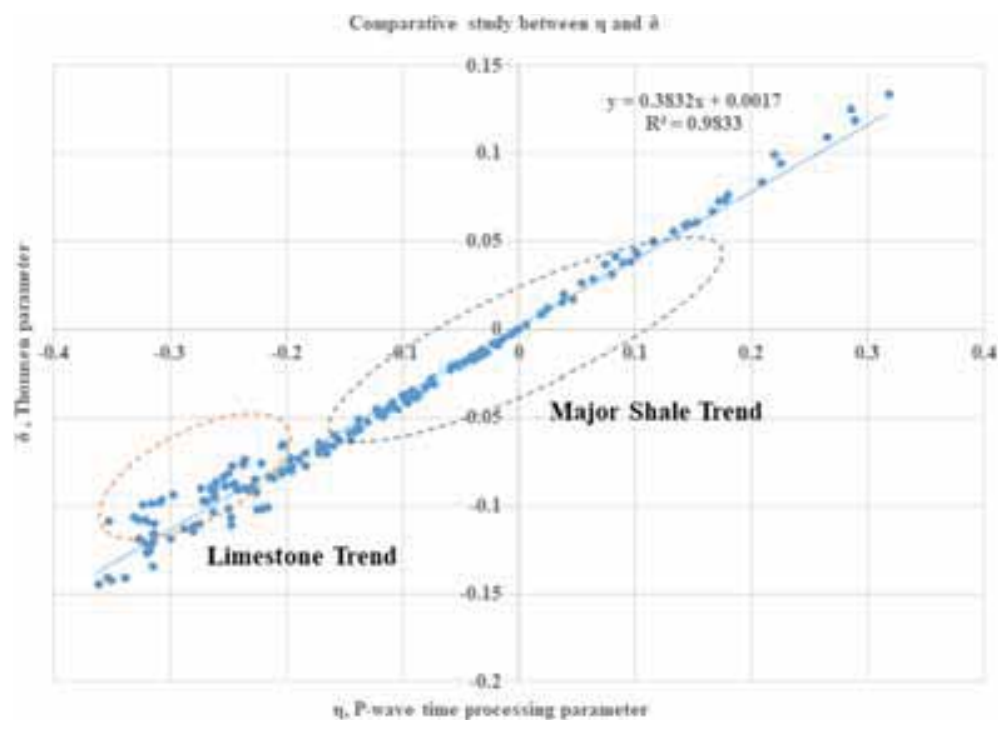

Figure 16. A comparative study between the Thomsen parameter $\delta$ and P-wave time processing parameter $\eta-$ shows lithology discrimination between a major shale trend and a limestone trend.

these two Thomsen parameters which strongly denote a permeable, as well as non-permeable section, present in the Goru formation. The positive value of $\epsilon$ and $\gamma$ shows good source rock content (TOC) of this study zone in Goru formation along with micro-fracture and layers of shale towards the representation of a strong anisotropy nature (Ross and Bustin 2008, 2009; Loucks et al. 2009; Ostadhassan et al. 2012). The negative value of $\epsilon$ and $\gamma$ shows the presence of the permeable section in the formation. In a few points, we observed that vertical velocity is faster compared to horizontal velocity as $\epsilon$ has a fractional difference between vertical and horizontal compressional wave velocity. In the case of $\gamma$ which is a horizontal S-wave and horizontal polarisation to the vertical S-wave, there is a faster vertical shear velocity than horizontal shear velocity (Thomsen 1986). We observed the presence of fluid in horizontal microlayers and fractures in the study section. Sometimes the presence of this fluid creates an obstacle for propagating the vertical wave; as a result, we observed a rapid increment of horizontal bedding velocity with depth in those regions especially for the diffraction event (Shipley et al. 1997; Ostadhassan et al. 2012). Petrophysical properties such as porosity are significant factors for enhancing anisotropy in the shale dominated section due to the compaction of the rock matrix structure. For a few samples of the study well A, the values of $\epsilon$ and $\gamma$ are small and quite minimum and these samples represent that the zone is in isotropic medium thus missing the permeable zone observed. However, most of the samples of the study well A show a consistent value of $\epsilon$ and $\gamma$. These evaluated results of Thomsen parameters show that the vertical transverse isotropic behaviour of shale, which represents the chances of a vertical fracture in this study formation for well $\mathrm{A}$, is negligible.

\section{Conclusions}

The goal of this study is to show the importance of the anisotropy factor for velocity analysis in the tectonically deformed hydrocarbon-bearing reservoir. This study shows that the use of anisotropy incorporated velocities provides a robust result towards finding new scopes for hydrocarbon accumulation in challenging geological reservoirs. In a few areas of Indian sedimentary basins, sufficient well log data are not available due to the unavailability of logging tools and old data acquiring techniques. This limitation produces a discouraging result in hydrocarbon exploration. The Ghotaru area of Jaisalmer sub-basin is also this kind of area where the availability of data is limited, and most of the acquired data are more than 30 yrs old. However, geologically, this area is a good prospect for hydrocarbon exploration. However, this area was not successful with respect to exploration and even the study well also produced an unproductive result. We observed a few grains of dissolved gas observed in this study well A during testing. This study represents that the anisotropy parameter incorporation in the non-reservoir shale section 
impacts the geological model to the full-scale which leads to producing a robust result for hydrocarbon exploration. The study shows an impactful and distinguished results for a further study in this area for finding hydrocarbons with excellent lithology discrimination based on anisotropy parameters incorporated velocity analysis. A robust study with proper techniques will produce a more encouraging result for hydrocarbon exploration in Ghotaru region.

\section{Acknowledgements}

The authors are thankful to NDR (National Data Repository), DGH (Director General of Hydrocarbon), India and GSPC, Gandhinagar, India for providing technical data support for research. The authors are profoundly thankful to Geophysical Data Quantitative Interpretation Lab. (GQIL), Department of Applied Geophysics, IIT(ISM) Dhanbad for providing support to carry out this research work. The authors would like to acknowledge M/s Schlumberger for providing R\&D license for the research work. We are obliged to the reviewers of the Journal of Earth System Science for their critical review of this paper.

\section{References}

Alkhalifah $\mathrm{T}$ and Tsvankin I 1995 Velocity analysis for transversely isotropic media; Geophysics 60 1550-1566.

Avseth P 2015 Explorational rock physics: The link between geological processes and geophysical observables; Petrol. Geosci., https://doi.org/10.1007/978-3642-02332-3_18.

Castagna J P, Batzle M L and Eastwood R L 1985 Relationships between compressional-wave and shear-wave velocities in clastic silicate rocks; Geophysics 50(4) 571-581.

Chesnokov E M, Tiwary D K, Bayuk I O, Sparkman M A and Brown R L 2009 Mathematical modelling of anisotropy of illite-rich shale; Geophys. J. Int. 178 1625-1648.

Datta Gupta S and Gupta R 2017 Importance of coloured inversion technique for thin hydrocarbon sand reservoir detection - A case in mid Cambay basin; J. Geol. Soc. India, Springer 90(4) 485-494.

Datta Gupta S, Chatterjee R and Farooqui M Y 2012 Rock physics template (RPT) analysis of well logs and seismic data for lithology and fluid classification in Cambay basin; Int. J. Earth Sci. 101(5) 1407-1426.

Dobson P J 1985 Physical properties of crystals - Their representation by tensors and matrices; Phys. Bull. 36(12) 506.

Far M E, Quirein A J and Mekic N 2015 Orthorhombic model for geomechanical applications in unconventional shale reservoirs; SEG Technical Program Expanded Abstracts.
Han D H, Nur A and Morgan D 1986 Effects of porosity and clay content on wave velocities in sandstone; Geophysics $\mathbf{5 1}$ 2093-2107.

Higgins S, Goodwin S, Donald Q, Donald A, Bratton T and Tracy G 2008 Anisotropic stress models improve completion design in the Baxter shale, Society of Petroleum Engineers (SPE)/115736; Annual Technical Conference and Exhibition, Denver, Colorado, USA.

Hornby B E 1994 The elastic properties of shales; PhD Thesis, University of Cambridge, Cambridge, Massachusetts, 201p.

Lee M W 2010 Predicting S-wave velocities for unconsolidated sediments at low effective pressure; U.S. Geological Survey Scientific Investigations Report, 2010-5138, 13p.

Loucks R G, Reed R M, Ruppel S C and Jarvie D M 2009 Morphology, genesis, and distribution of nanometer-scale pores in siliceous mudstones of the Mississippian Barnett Shale; J. Sedim. Res. 79 848-861.

Mavko G, Mukerji T and Dvorkin J 2009 The rock physics handbook; Cambridge University Press, Cambridge.

Norris A and Sinha B 1993 Weak elastic anisotropy and the tube wave; Geophysics 58 1091-1098.

Nye J F 1985 Physical properties of crystals: Their representations by tensors and matrices; Oxford University Press, Clarendon Press, New York, 329p.

Okorie I P C, Ebeniro J O and Ehirim C N 2016 Anisotropy and empirical relations for the estimation of anisotropy parameters in Niger Delta depobelts; Int. J. Geosci. 7 $345-352$.

Ostadhassan M, Zeng Z and Jabbari H 2012 Anisotropy analysis in shale using advanced sonic data - Bakken case study; $A A P G$ Bull., Annual Convention and Exhibition, Search and Discovery Article.

Prasad M 2002 Acoustic measurements in unconsolidated sands at low effective pressure and overpressure detection; Geophysics 67 405-412.

Prasad M and Nur A 2003 Velocity and attenuation anisotropy in reservoir rocks, Society of Exploration Geophysicists (SEG) Technical program, Dallas, Texas.

Ross D J K and Bustin R M 2008 Characterizing the shale gas resource potential of Devonian-Mississippian strata in the Western Canada sedimentary basin: Application of an integrated formation evaluation; AAPG Bull. 92 87-125.

Ross D J K and Bustin R M 2009 The importance of shale composition and pore structure upon gas storage potential of shale gas reservoirs; Mar. Pet. Geol. 26 916-927.

Ruger A 1998 Variation of P-wave reflectivity with offset and azimuth in anisotropic media; Geophysics 63 935-947.

Ruger A 2002 Reflection coefficients and azimuthal AVO analysis in anisotropic media; Society of exploration geophysicists (SEG), Tulsa.

Sayers C M 1994 The elastic anisotropy of shales; J. Geophys. Res.-Sol. EA 99 767-774.

Sayers C M 2005 Seismic anisotropy of shales; Geophysics 64 93-98.

Sharma K K 2007 K-T magmatism and basin tectonism in western Rajasthan, India, results from extensional tectonics and not from reunion plume activity; Geol. Soc. Am., https://doi.org/10.1130/2007.2430(35).

Shipley T H, Ogawa Y, Blum P, Bahr J M, Bruckmann W, Moran K and Housen B A 1997 Directional properties of $\mathrm{P}$-wave velocities and acoustic anisotropy in different structural domain of the Northern Barbadose ridge 
Accretionary complex; In: Proceedings of the ocean drilling program, scientific results, $156 \mathrm{p}$.

Shoenberg M, Muir F and Sayers C M 1996 Introducing ANNIE: A simple three parameters anisotropic velocity model for shales; J. Seism. Explor. 5 35-49.

Simm R and Bacon M 2014 Seismic amplitude: An interpreter's handbook; Cambridge University Press, Cambridge, $84 \mathrm{p}$.

Tatham R H and McCormack M D 1991 Multicomponent seismology in petroleum exploration; Society of Exploration Geophysicists (SEG), Tulsa.

Thomsen L 1986 Weak elastic anisotropy; Geophysics 51(10) 1954-1966.

Corresponding editor: N V Chalapathi RaO
Tsvankin I 1997 Seismic signatures and analysis of reflection data in anisotropic media (1st edn); Elsevier Science, Amsterdam, 29p.

Tsvankin I 2005 Seismic signatures and analysis of reflection data in anisotropic media (2nd edn); Elsevier Science, Amsterdam.

Vernik L L and Liu X 1997 Velocity anisotropy in shales - A petrophysical study; Geophysics 62 521-532.

Walsh J, Sinha B and Donald A 2006 Formation anisotropy parameters using borehole sonic data, Society of Petrophysicists and Well Log Analysts (SPWLA) 47th annual logging symposium, Veracruz, Mexico.

Zimmer M A 2003 Seismic velocities in unconsolidated sands Measurements of pressure, sorting, and compaction effects; PhD Thesis, Palo Alto, Calif., Stanford, 204p. 